\title{
Advancing the Prevention of Substance Abuse via Translational Research
}

\author{
Diana Fishbein, PhD
}

Estimates of the total costs of substance abuse in the United States, including productivity and health- and crime-related costs, exceed $\$ 600$ billion annually (approximately $\$ 181$ billion for illicit drugs, $\$ 193$ billion for tobacco, and $\$ 235$ billion for alcohol). Twenty-five percent of all US deaths can be attributed to drug abuse. Alcohol, the most commonly used drug among American teenagers, kills youth 6.5 times more often than other drugs of abuse. In general, a yearly death toll of nearly 10,000 can be attributed to chronic alcohol abuse. Drug consumption of all types generally begins in adolescence; by 13 years of age more than $30 \%$ of teens report having used at least one illicit substance. As staggering as these numbers are, they do not fully describe the breadth of corollary destructive public health and safety implications, such as family disintegration, loss of employment, failure in school, domestic violence, and child abuse.

Hundreds of millions of research dollars have been spent on preventive intervention programs aimed at curbing drug abuse and addiction. At the National Institute of Health alone, more than $\$ 1.6$ billion was spent in 2010 on substance abuse, exceeding expenditures for any other neurobehavioral disease. Despite this enormous amount of scholarly effort, most prevention research has neither focused on nor sought to ameliorate generative and oftentimes malleable underlying mechanisms (e.g., neurocognition) in drug abuse and addiction or the related psychopathology. A significant problem is the lack of communication among scientists investigating disparate aspects of the substance abuse issue. Transfer of information between disciplines has been slow; no major innovations in prevention have occurred for decades. As a result, although some recipients of preventive interventions (e.g., school-based programs) may achieve some measure of success, many others respond less favorably, exhibiting an escalation of drug-related disorders, chronic relapses, lack of engagement in interventions, and persistent intervention resistance. Moreover, policy makers and practitioners lack the knowledge base and resources to design programs that

\section{Key Research Opportunities}

Translational research in substance abuse conducted by transdisciplinary teams affords better opportunities to parse the complex web of factors affecting successful prevention by

- Elucidating underlying mechanisms through neurobiological and contextual research that integrates multiple domains of the process

- Subtyping individuals based on intervention responsivity by assessing biological, physical, social, and community factors

- Charting individual- and group-level change in response to interventions and social conditions by fully characterizing multiple dimensions of behavioral outcomes

- Fostering analytic innovations to address and integrate the multiple dimensions.

Investment in this line of research has potential to advance the field of substance abuse and lead to significant advancements in the prevention of the global substance abuse problem.

can have meaningful impacts. Forming teams composed of investigators from multiple disciplines that span the pathway from basic science to applied research and practice will facilitate the translation of findings to the next phase of inquiry, thus promoting a more rapid response to these issues than has been achieved in the past.

This research brief highlights the relevance and utility of promoting a transdisciplinary translational model as the foundation for the next generation of research on substance abuse, including tobacco, alcohol, illicit drugs, and illegitimate use of prescription drugs. The model integrates theoretical perspectives and empirical methods to (1) elucidate the 
causes of substance abuse; (2) identify malleable targets (e.g., cognitive function, socio-emotional skills, child maltreatment) for intervention; (3) yield a better understanding of how preventive interventions work (or do not work) for individuals or subgroups; (4) develop, refine, and evaluate targeted interventions and policies; and (5) disseminate and deliver indicated programs and services. The model connects the dots across the translational pathway to successfully address the full spectrum of issues that influence individuals as either risk or protective factors in the development of substance abuse.

Collaborations that cross disciplinary and organizational boundaries will lead to a greater understanding of how neurogenetic mechanisms (e.g., neurotransmitter imbalance, psychiatric disturbance, cognitive deficits) interact with environmental factors (such as family dysfunction, witnessing violence, poverty) to influence substance use initiation, escalation, and transitions to abuse and dependence. Furthermore, cross-cutting work is likely to help identify factors that promote or interfere with improvements in behavior in response to preventive interventions. Fostering tangible interactions between basic and applied scientists, and institutionalizing a research architecture comprising multiple theoretical and methodological approaches, can lead to the development of novel ideas and innovative approaches that may advance our knowledge more rapidly.

\section{Characteristics of Transdisciplinary Research}

For research to be truly transdisciplinary, various disciplinary perspectives and approaches must be woven into an integrated framework rather than left as a compartmentalized set of capabilities. The continuum of integrated scientific research includes the following, somewhat distinctive, graduated categories:

- multidisciplinary research, in which investigators from different disciplines work independently, and largely sequentially, on a given research issue with the goal of combining their work into a single research product;

- interdisciplinary research, in which investigators from different disciplines work jointly, with each bringing their own discipline's perspective to key issues; and

- transdisciplinary research, in which investigators transcend the methods and theories of their disciplines by jointly developing integrative methodology and theory.

The high degree of synthesis and integration afforded by transdisciplinary research offers great potential to generate innovative solutions to society's varied pressing problems, such as substance abuse. Conducting transdisciplinary research requires considerable organizational and fiscal resources, a common lexicon, and shared methods. For transdisciplinary research to succeed, a deliberate and vigorous investment of intellectual, fiscal, and personnel resources is needed to transform multidisciplinary and interdisciplinary approaches into a true transdisciplinary framework.

\section{Translating Basic Research to Applied Scientific Questions}

Translational research is the process of applying discoveries generated during research in the laboratory and in preclinical studies to the development of trials and studies in humans; it is also the process of applying bedside observations to inform benchtop discoveries. ${ }^{1}$ It aims to enhance adoption of best practices in the community and to contribute to eventual applications at a later point along the "translational pathway." Translational research is inherently transdisciplinary given the reliance on multiple perspectives and approaches to advance the research to the next stage of inquiry and application. Figure 1 provides definitions of translation (Types 1, 2, and 3) and highlights areas of inquiry that constitute each phase in a drug abuse prevention model.

Toward this end, NIH is proposing to establish the National Center for Advancing Translational Science (NCATS), designed to revolutionize the science of translation and catalyze the transfer of knowledge across this pathway for all diseases. $^{2}$

\section{The End Game: Personalized Prevention}

A new generation of transdisciplinary neuroscience research has established that individual differences in risk for substance abuse can be thoroughly understood only by recognizing that human behavior relies largely on genetic and neurobiological mechanisms that are sculpted by numerous socio-

environmental factors (Figure 1). Interactions among these underlying mechanisms and exposures to nurturing or adverse environments will bias developmental trajectories toward favorable or psychopathological outcomes, respectively.

For example, evidence suggests that certain genetic variants may increase risk for substance abuse, ${ }^{3}$ possibly through alterations in decision-making ability, novelty-seeking, and other cognitive and behavioral traits. ${ }^{4}$ Additionally, environmental factors such as stress confer differential risks that are contingent on underlying genetic variants. ${ }^{5}$ Importantly, neural dysfunctions that often underlie behavioral problems such as substance abuse are often preventable and to some extent modifiable. Programs targeted to underlying dysfunctions may strengthen or compensate for cognitive and emotional regulatory processes (and their neural substrates) that often accompany and antedate substance abuse. Such 


\section{Figure 1. Factors in the translational drug abuse prevention model}

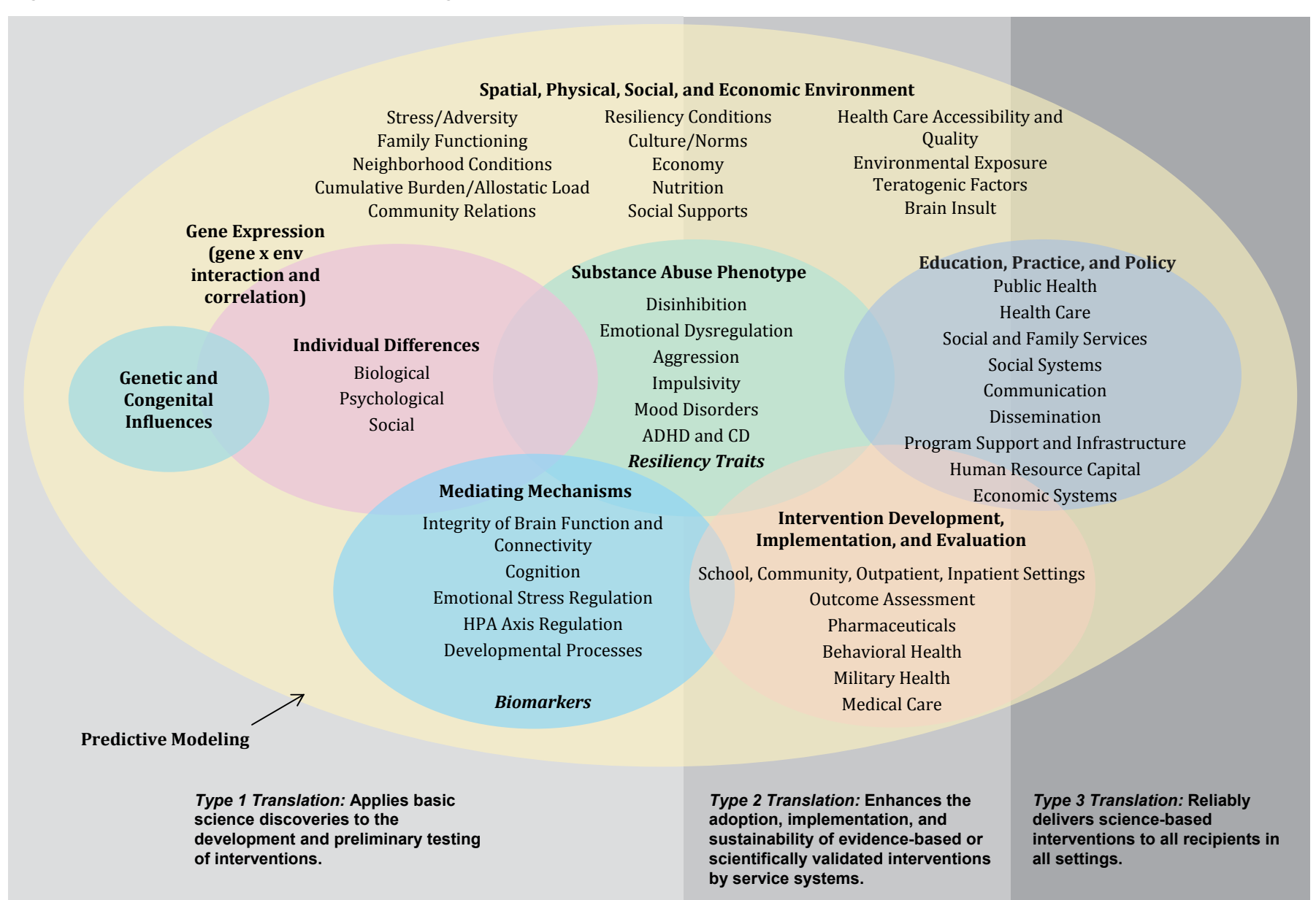

$\mathrm{ADHD}=$ Attention Deficit/Hyperactivity Disorder; $\mathrm{CD}=$ Conduct Disorder; gene $\mathrm{x}$ env = gene by environmental interactions;

$\mathrm{HPA}=$ hypothalamic-pituitary-adrenal.

findings have direct implications for designing interventions to prevent substance abuse; thus, they have extraordinary potential significance for mental and public health policies.

The ultimate question for designing a personalized approach to prevent the development of substance abuse is "what works best, for whom, why, and under what circumstances?" Four strategies facilitate this translational process:

1. Identifying underlying conditions that increase risk for substance abuse (e.g., attention deficit, conduct disorder, depression, novelty-seeking, adversity) and applying this knowledge to better understand people's differential responsiveness to preventive interventions. ${ }^{6}$ Although our knowledge of such mechanisms has grown appreciably in recent years, the clinical utility and appeal of even "successful" programs are both limited in the eyes of practitioners and policy makers. Factors underlying both favorable and poor responses remain largely unknown because of a lack of translation from bench science to intervention.
2. Characterizing individuals based on factors that underlie their behavioral problems and subsequently evaluating intervention outcomes by stratifying on those characteristics. Conventional approaches group study participants on the basis of experimental and control assignments and, in some cases, stratify on sex, sociodemographic, or psychological characteristics that are not amenable to change. The result is that such groups comprise individuals with diverse but highly relevant underlying characteristics, which dilutes estimated effect sizes and detracts from identifying individual-level factors that promote or interfere with favorable outcomes. The suggested approach instead decomposes the groups to construct more specific stratifications that are informed by a priori knowledge from basic science findings on the generators of behavioral problems and distinguished by varying levels of intervention responsivity. It then becomes possible to determine what works best for whom and why. 
3. Charting changes in underlying conditions (e.g., neurocognitive and emotional regulatory functional deficits) in response to an intervention. We anticipate that favorable outcomes would be accompanied by improvements in underlying mediators and that poor outcomes would be denoted by relatively small or no changes. A better understanding of the factors that influence outcome variability at the individual and subgroup levels will lead to a new generation of interventions more effectively targeted to individuals (or subgroups) who are less amenable to conventional approaches.

4. Developing novel methods to analyze data from existing and new datasets (generated by both clinical trials and observational studies) to highlight how underlying mechanisms correlate and interact to promote or interfere with improvements in substance abuse-related behavioral outcomes in response to interventions. For example, a latent class analysis approach can measure change within and between subtypes by identifying a small set of subgroups characterized by multiple dimensions that investigators can then examine to determine differential treatment effects. ${ }^{7}$ This approach minimizes methodological challenges that often occur in subgroup analysis, including a high rate of false positives, low statistical power, and limitations in examining higher-order interactions.

By recognizing multiple theoretical and methodological levels (i.e., from neurological to social and environmental) and using multiple analytic strategies (e.g., path or connectivity analyses), these approaches will facilitate targeting intervention resources to subgroups that promise to show the maximum intervention response.

\section{Conclusion}

The key implication of this program of research is that tailored, targeted interventions will be most effective when psychosocial programs are matched to the individual's or group's constellation of social, environmental, psychological, and biological attributes. This approach will reinforce adaptive behavioral responses, compensatory systems, and inhibitory mechanisms. Development, implementation, and refinement of the resulting science-based interventions in different populations and settings can then lead back to the etiological sciences (backward translation) in a cyclic way, resulting in better understanding of the mechanisms underlying poor therapeutic outcomes among other subgroups. The ultimate goal is that, through a transfer of knowledge between science and practice, public health strategies and policies will become increasingly responsive and appropriate, thereby exerting a more global impact than has been the case.

\section{References}

1. Dougherty D, Conway PH. The "3T's" road map to transform US health care: the "how" of high-quality care. JAMA. 2008;299(19):2319-21.

2. Collins, F. Reengineering translational science: the time is right. Sci Transl Med. 2011 Jul 6;3(90):90cm17,1-6. doi: 10.1126/ scitranslmed.3002747.

3. Le Foll B, Gallo A, Le Strat, Y, Lu L, Gorwood P. Genetics of dopamine receptors and drug addiction: a comprehensive review. Behav Pharmacol 2009;20(1):1-17.

4. Lusher JM, Chandler C, Ball D. Dopamine D4 receptor gene (DRD4) is associated with Novelty Seeking (NS) and substance abuse: the saga continues ... Mol Psychiatry. 2001;6(5):497-9.

5. Enoch MA. The role of early life stress as a predictor for alcohol and drug dependence. Psychopharmacology (Berl). 2011;214(1):17-31.

6. Fishbein DH, Hyde C, Eldreth D, Paschall MJ, Tarter R, Das A, et al. Neurocognitive skills moderate urban male adolescents' responses to preventive intervention materials. Drug Alcohol Depend. 2006 Mar 15;82(1):47-60. Epub 2005 Sep 8.

7. Lanza ST, Rhoades BL. Latent class analysis: an alternative perspective on subgroup analysis in prevention and treatment. Prev Sci. 2011 Feb 12. [Epub ahead of print]

\footnotetext{
About the Author

Diana H. Fishbein, a behavioral neuroscientist, is a Senior Fellow at RTI International and directs the Transdisciplinary Science and Translational Prevention Program in RTI's Molecular Epidemiology, Genomics, Environment and Health (MEGEH) Center.

RTI Press Research Briefs and Policy Briefs are scholarly essays on policy, methods, or other topics relevant to RTI areas of research or technical focus.

RTI International, 3040 Cornwallis Road, PO Box 12194 Research Triangle Park, NC 27709-2194 USA

$919.541 .6000 \quad$ rtipress@rti.org www.rti.org

O2011 Research Triangle Institute. RTI International is a trade name of Research Triangle Institute.

All rights reserved. Please note that this document is copyrighted and credit must be provided to the authors and source of the document when you quote from it. You must not sell the document or make a profit from reproducing it.

RTI Press publication RB-0003-1108

doi:10.3768/rtipress.2011.rb.0003.1108

www.rti.org/rtipress
} 